\title{
Changes in the Incidence and Etiological Patterns of Bacteremia Associated with Acute Leukemia over a 25-Year Period
}

\author{
Hisashi FunadA and Tamotsu MATsudA*
}

\begin{abstract}
During the 25-year period from 1972-1996, 360 episodes of bacteremia occurred in $248(45 \%)$ of a total of 548 patients with acute leukemia on our hematology ward, with the rate of occurrence remaining almost unchanged. Patients with acute nonlymphocytic leukemia, however, had a significant decrease in the incidence of bacteremia during the last 5-year period. Gram-negative bacilli decreased in relative frequency from $64 \%$ for the first 15 -year period to $40 \%$ for the last 5 year period, whereas gram-positive cocci increased from $24 \%$ to $51 \%$. Escherichia coli and Enterobacter cloacae somewhat decreased in frequency, whereas coagulase-negative staphylococci and streptococci had considerable increases. In contrast, Pseudomonas aeruginosa was isolated at a relatively constant frequency, forming one of the top two pathogens throughout the study period. Despite the shift toward gram-positive cocci, therefore, $P$. aeruginosa remains a key organism in considering the initial empiric antibiotic regimen and infection prevention during neutropenia. (Internal Medicine 37: 1014-1018, 1998)
\end{abstract}

Key words: gram-positive cocci, gram-negative bacilli, empiric antibiotic therapy, Pseudomonas aeruginosa

\section{Introduction}

Bacteremia remains a frequent complication with serious morbidity and mortality in patients with acute leukemia undergoing intensive chemotherapy and/or hematopoietic stem cell transplantation $(1,2)$. In many cancer centers, gram-positive cocci, mostly coagulase-negative staphylococci (CNS), now have virtually replaced enteric gram-negative bacilli as major pathogens $(3,4)$. However, gram-negative bacteremia, particularly due to Pseudomonas aeruginosa still has a poor prognosis $(5,6)$. Thus, the prevalence patterns for bacteremia pathogens play an important role in the choice of antibiotics for the initial empiric treatment of febrile patients with cancer.

The present study reviews our experience with bacteremia complicating acute leukemia over the 25 years from 1972 1996 , focusing on both the recent decrease in the frequency of bacteremia seen among patients with acute nonlymphocytic leukemia (ANLL) and the clinical significance of $P$. aeruginosa in the face of preponderant gram-positive cocci.

\section{Patients and Methods}

\section{Patients}

A total of 548 patients with acute leukemia [434 with ANLL and 114 with acute lymphocytic leukemia (ALL)] were admitted to and discharged from the Third Department of Medicine, Kanazawa University Hospital to undergo intensive chemotherapy and/or hematopoietic stem cell transplantation on 773 occasions during the 25-year period, 1972-1996. Three hundred and thirteen of the patients were males and 235 females, with a median age of 45 (range, 8-86) years. The patients underwent intensive induction or reinduction treatment and, after achieving a complete remission, further intense treatment referred to as consolidation or maintenance/intensification therapy. The first 15-year period, 1972-1986, was described elsewhere (1) and is included for purposes of comparison.

During the 25-year period, supportive measures for neutropenic patients with cancer changed considerably with the availability of antifungal prophylaxis, effective empiric antibiotic and antifungal therapy, central venous catheters, and recombinant human granulocyte colony-stimulating factor (rhG-

From the Department of Infectious Prophylaxis, Faculty of Medicine, Toyama Medical and Pharmaceutical University, Toyama and *the Third Department of Medicine, Faculty of Medicine, Kanazawa University, Kanazawa

Received for publication January 9, 1998; Accepted for publication August 24, 1998

Reprint requests should be addressed to Dr. Hisashi Funada, the Department of Infectious Prophylaxis, Faculty of Medicine, Toyama Medical and Pharmaceutical University, 2630 Sugitani, Toyama 930-0194 


\section{Bacteremia in Acute Leukemia}

I . Oral antifungal prophylaxis NYS or AMPH

1972

II. Empiric antibiotic therapy

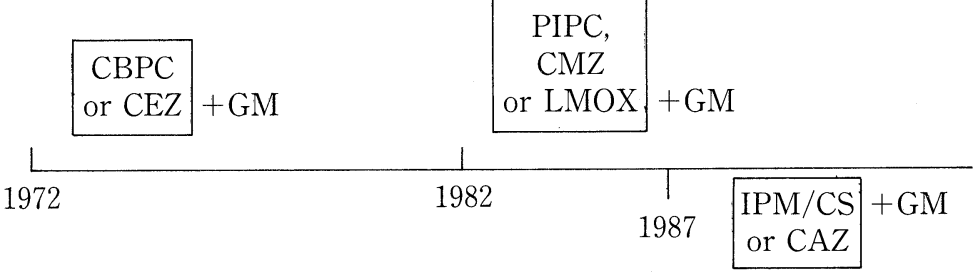

III. Empiric antifungal therapy

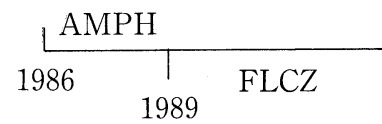

IV. Use of rhG-CSF

V. Central venous catheters

Figure 1. Changes in supportive measures for neutropenic cancer patients. AMPH: amphotericin B, CAZ: ceftazidime, CBPC: carbenicillin, CEZ: cefazolin, CMZ: cefmetazole, FLCZ: fluconazole, GM: gentamicin, IPM/ CS: imipenem/cilastatin, LMOX: latamoxef, NYS: nystatin, PIPC: piperacillin, rhG-CSF: recombinant human granulocyte colony-stimulating factor.

CSF) (Fig. 1). The rhG-CSF was administered mainly to patients with ALL as well as those with relapsed or refractory acute leukemia (7). All but transplant patients were treated on one 40-bed hospital floor and exposed to similar potential environmental contaminants under routine hospital care. Transplant patients received total or selective intestinal decontamination in protected environments, often combined with rhG-CSF prophylaxis, until posttransplant neutrophil counts exceeded $500 / \mu l(8,9)$.

\section{Blood cultures}

Blood cultures were described elsewhere (1). Briefly, blood was obtained by venipuncture or through catheter. Culture Bottles Nos. 1 and 2 (Eiken) (brain-heart infusion broth and thioglycollate medium, respectively) were replaced in 1982 by Liquoid Blood Culture Bottles TSB and Thio (Roche) (tryptic soy both and thioglycollate medium, respectively).

\section{Bacteremia}

The diagnosis of bacteremia was based on at least one positive blood culture from a patient in a consistent clinical setting. Polymicrobial bacteremia was defined as the concurrent or sequential recovery of two or more different organisms during any 1 -week period. Fungemia was expediently included in the category of bacteremia, since Candida was the only fungal organism that was isolated from $12(3 \%)$ of all 360 bloodstream infections.

\section{Statistics}

Statistical analyses were performed using the chi-square test with Yates' correction (in conjunction with Fisher's exact test where appropriate).

\section{Results}

\section{Incidence of bacteremia}

During a 25-year period, 360 episodes of bacteremia occurred in $248(45 \%)$ of a total of 548 patients with acute leukemia [193 (44\%) of 434 with ANLL and 55 (48\%) of 114 with ALL], which gives an average of 1.5 episodes per patient. Seventy-eight patients $(31 \%)$ experienced multiple episodes (53 with two episodes, 19 with three episodes, and six with four or more episodes). The median interval between episodes was 59 days, with a range of 10 days to 29 months. Polymicrobial bacteremia was seen in 45 episodes (13\%), comprising 25 of the concurrent type and 20 of the sequential type. The overall incidence of bacteremia was 466 episodes per 1,000 hospital admissions (360 episodes occurring during 773 periods in hospital). The distribution of patients with bacteremia by age, sex and leukemic type did not differ materially from that of the total patient population with acute leukemia.

The incidence of bacteremia was compared between the first 15-year period, 1972-1986 and the last 5-year period, 19921996 (Table 1). The two patient groups were similar with regard to age, sex and leukemic type. The percentage of patients with bacteremia in the last period was slightly but not significantly lower than that in the first period $[37 \%(50 / 136)$ versus $45 \%$ $\left.(132 / 291), X^{2}=2.460,0.10<p<0.20\right]$. On the other hand, the incidence of bacteremia per 1,000 hospital admissions decreased by 108 episodes (a $23 \%$ reduction) between the two periods (370 episodes for the last period compared with 478 episodes for the first period). Among the patients with ANLL, however, the incidence of bacteremia in the last period was significantly lower than that in the first period $[32 \%(36 / 111)$ 
Table 1. Frequency of Bacteremia in Patients with Acute Leukemia

\begin{tabular}{lcc}
\hline & \multicolumn{2}{c}{ Study period, years } \\
\cline { 2 - 3 } Data & $1972-1986$ & $1992-1996$ \\
\hline ALL & 291 & 136 \\
Total no. of patients admitted* & 229 & 111 \\
Underlying disease, no. ANLL & 62 & 25 \\
& 406 & 200 \\
Total no. of periods in hospital & $132(45)$ & $50(37)$ \\
No. (\%) of patients with bacteremia & $42(9-81)$ & $45(15-74)$ \\
Age, years Median (Range) & $74 / 58$ & $30 / 20$ \\
Sex, no. Male/Female & $106(46)$ & $36(32)$ \\
Underlying disease, no. (\%) ANLL & $26(42)$ & $14(56)$ \\
& 194 & 74 \\
No. of episodes of bacteremia & 1.5 & 1.5 \\
No. of episodes per patient & & \\
No. (\%) of episodes of polymicrobial & $28(14)$ & $3(4)$ \\
bacteremia & & \\
No. of episodes per 1,000 hospital & 478 & 370 \\
admissions &
\end{tabular}

*Patients with multiple periods in hospital or multiple episodes of bacteremia are included only once with regard to each study period. ANLL: acute nonlymphocytic leukemia, ALL: acute lymphocytic leukemia.

versus $46 \%$ (106/229), $\left.\mathrm{X}^{2}=5.345, \mathrm{p}<0.05\right]$, although such a decrease in incidence was not observed among the patients with ALL [56\% (14/25) for the last period compared with $42 \%$ (26/ 62) for the first period]. Moreover, the rate of polymicrobial bacteremia decreased significantly from $14 \%(28 / 194)$ in the first period to $4 \%(3 / 74)$ in the last period ( $\mathrm{p}<0.05$ by Fisher's exact test). Indeed, 23 (74\%) of the 31 polymicrobial episodes occurring in both periods were associated with ANLL.

\section{Causative organisms}

A total of 413 blood culture isolates were obtained from all 360 episodes of bacteremia over the 25 years (Table 2). Aerobic gram-negative bacilli (56\%) were the most frequent causative organisms, followed in frequency by aerobic gram-positive cocci (31\%), anaerobes (8\%), Candida species (3\%) and aerobic gram-positive bacilli (2\%). According to the individual species or groups of organisms, $P$. aeruginosa $(17 \%)$ was the most common organism, followed in frequency by Escherichia coli (15\%), CNS (13\%), enterococci (9\%), Klebsiella pneumoniae (8\%) and Enterobacter cloacae (7\%). However, their relative frequency considerably changed between the first 15-year period, 1972-1986 and the last 5-year period, 19921996, with an intermediate pattern of frequency seen in the second 5-year period, 1987-1991.

The relative frequency of gram-negative bacilli decreased significantly from $64 \%$ (147/228) for the first period to $40 \%$ (31/77) for the last period $\left(X^{2}=12.909, p<0.01\right)$. In contrast, gram-positive cocci showed a marked increase in frequency from $24 \%(54 / 228)$ to $51 \%(39 / 77)\left(X^{2}=18.496, p<0.01\right)$.

The top five pathogens found during the first period (in descending order of frequency) were $E$. coli, $P$. aeruginosa, CNS, K. pneumoniae and E. cloacae, of which all but one are gram-negative bacilli. However, the relative frequency of $E$. coli decreased slightly but not significantly from $21 \%(47 / 228)$ in the first period to $10 \%(8 / 77)$ in the last period $\left(\mathrm{X}^{2}=3.409\right.$, $0.05<\mathrm{p}<0.10)$. E. cloacae also showed a similar decrease in frequency from $10 \%(22 / 228)$ to $4 \%(3 / 77)(0.05<\mathrm{p}<0.10$ by Fisher's exact test). In contrast, $P$. aeruginos $a$ was isolated at about the same frequency during the first and last periods [15\% (35/228) and 14\% (11/77), respectively], although the organism ranked first in frequency [21\% (23/108)] in the second period; there was, however, no significant difference in frequency among the three periods $\left(\mathrm{X}^{2}=1.707,0.30<\mathrm{p}<0.50\right) . K$. pneumoniae also showed a similar trend in frequency [10\% (23/ 228 ) and $8 \%$ (6/77), respectively, for the first and last periods].

Gram-positive cocci showed a tendency to increase in frequency throughout the study period. The relative frequency of CNS increased significantly from $11 \%(25 / 228)$ for the first period to $23 \%(18 / 77)$ for the last period $\left(\mathrm{X}^{2}=6.333, \mathrm{p}<0.05\right)$, and this group of organisms came to the top ahead of $P$. aeruginosa. Streptococci also increased in frequency from $2 \%$ $(5 / 228)$ to $9 \%(7 / 77)\left(\mathrm{X}^{2}=5.536, \mathrm{p}<0.05\right)$. Moreover, enterococci were isolated at the same frequency as E. coli $[10 \%(8 / 77)$ each] during the last period. Thus, the top five pathogens found during the last period included enterococci and streptococci, both of which replaced K. pneumoniae and E. cloacae. Among the enterococci, Enterococcus faecium was more frequently isolated than Enterococcus faecalis. S. aureus, although in small numbers, was also isolated at an increasing frequency, but most of the isolates proved to be methicillin-sensitive.

\section{Discussion}

Recent data from a Centers for Disease Control and Prevention survey disclosed that the percentage of primary bacteremia among all nosocomial infections increased from $7 \%$ between 1980 and 1986 to $13 \%$ between 1990 and 1992 (10). In contrast, the present study showed that the percentage of patients with acute leukemia who developed bacteremia remained almost unchanged during the past 25 years, although the incidence of bacteremia per 1,000 hospital admissions decreased by 108 episodes. This reduction in incidence cannot easily be disregarded, however, since the average incidence per 1,000 hospital admissions in city or university hospitals has been reported as about 10 (range, 3.4-39) episodes (1).

Patients with ANLL had a significant decrease in the incidence of bacteremia in the last 5-year period, as contrasted with patients with ALL. This may reflect the fact that recent progress in the treatment of ANLL has somewhat surpassed that in the management of ALL, particularly in adult patients $(11,12)$. In 1992, the principle was set up that prednisolone should not be used for the treatment of ANLL in the AML-92 protocol of the Japan Adult Leukemia Study Group (12), which may have contributed to such a decrease in incidence. Moreover, the recent decrease in the rate of polymicrobial bacteremia was noted in the present series. This may be related to the fact that 
Bacteremia in Acute Leukemia

Table 2. Changing Patterns of Blood Culture Isolates from Patients with Acute Leukemia

\begin{tabular}{|c|c|c|c|c|c|c|c|c|}
\hline \multirow{3}{*}{ Organism } & \multicolumn{6}{|c|}{ Study period, years } & & \\
\hline & \multicolumn{2}{|c|}{$\begin{array}{c}1972-1986 \\
\text { (194 episodes) }\end{array}$} & \multicolumn{2}{|c|}{$\begin{array}{c}\text { 1987-1991 } \\
\text { (92 episodes) }\end{array}$} & \multicolumn{2}{|c|}{$\begin{array}{c}1992-1996 \\
\text { (74 episodes) }\end{array}$} & \multicolumn{2}{|c|}{$\begin{array}{c}\text { Total } \\
\text { (360 episodes) }\end{array}$} \\
\hline & $\begin{array}{l}\text { No. of } \\
\text { isolates }\end{array}$ & $\begin{array}{c}\text { Subtotal } \\
\text { no. }(\%)\end{array}$ & $\begin{array}{l}\text { No. of } \\
\text { isolates }\end{array}$ & $\begin{array}{c}\text { Subtotal } \\
\text { no. }(\%)\end{array}$ & $\begin{array}{l}\text { No. of } \\
\text { isolates }\end{array}$ & $\begin{array}{c}\text { Subtotal } \\
\text { no. }(\%)\end{array}$ & $\begin{array}{l}\text { No. of } \\
\text { isolates }\end{array}$ & $\begin{array}{c}\text { Subtotal } \\
\text { no. }(\%)\end{array}$ \\
\hline Escherichia coli & 47 & & 9 & & 8 & & 64 & \\
\hline Citrobacter freundii & 1 & & 1 & & & & 2 & \\
\hline Klebsiella pneumoniae & 23 & & 3 & & 6 & & 32 & \\
\hline Enterobacter cloacae & 22 & & 3 & & 3 & & 28 & \\
\hline Serratia marcescens & 3 & & 1 & & & & 4 & \\
\hline Pantoea agglomerans & & & 1 & & & & 1 & \\
\hline Edwardsiella tarda & & $147(64)$ & 1 & $53(49)$ & & $31(40)$ & 1 & $231(56)$ \\
\hline Salmonella enterica & 1 & & & & & & 1 & \\
\hline Pseudomonas aeruginosa & 35 & & 23 & & 11 & & 69 & \\
\hline Burkholderia cepacia & 1 & & 1 & & & & 2 & \\
\hline Stenotrophomonas maltophilia & & & 2 & & 1 & & 3 & \\
\hline Aeromonas hydrophila & 11 & & 4 & & & & 15 & \\
\hline Alcaligenes xylosoxidans & 3 & & & & 1 & & 4 & \\
\hline Acinetobacter calcoaceticus & & & 1 & & 1 & & 2 & \\
\hline Capnocytophaga sputigena & & & 3 & & & & 3 & \\
\hline Staphylococcus aureus & 9 & & 5 & & 6 & & 20 & \\
\hline Methicillin-resistant & 1 & & 4 & & 1 & & 6 & \\
\hline Coagulase-negative staphylococci & 25 & & 12 & & 18 & & 55 & \\
\hline Enterococcus species & 15 & $54(24)$ & 14 & $36(33)$ & 8 & $39(51)$ & 37 & $129(31)$ \\
\hline E. faecalis & 8 & & 6 & & 2 & & 16 & \\
\hline E. faecium & 7 & & 7 & & 5 & & 19 & \\
\hline E. avium & & & 1 & & 1 & & 2 & \\
\hline Streptococcus species & 5 & & 5 & & 7 & & 17 & \\
\hline S. pneumoniae & 1 & & & & 1 & & 2 & \\
\hline S. pyogenes & 1 & & & & & & 1 & \\
\hline Bacillus cereus & 3 & & 1 & & & & 4 & \\
\hline Lactobacillus species & & $5(2)$ & & $3(3)$ & 1 & $1(1)$ & 1 & $9(2)$ \\
\hline Corynebacterium species & 2 & & 2 & & & & 4 & \\
\hline Bacteroidaceae & 11 & & 8 & & 2 & & 21 & \\
\hline Bacteroides fragilis group & 4 & & 4 & & 2 & & 10 & \\
\hline Fusobacterium nucleatum & 2 & & 3 & & & & 5 & \\
\hline Propionibacterium acnes & 2 & $17(7)$ & 3 & $12(11)$ & 1 & $3(4)$ & 6 & $32(8)$ \\
\hline Clostridium perfringens & 4 & & 1 & & & & 5 & \\
\hline Candida species & 5 & $5(2)$ & 4 & $4(4)$ & 3 & $3(4)$ & 12 & $12(3)$ \\
\hline C. albicans & 1 & & 2 & & 2 & & 5 & \\
\hline Total & 228 & & 108 & & 77 & & 413 & \\
\hline
\end{tabular}

most of the polymicrobial cases occurred in patients with ANLL.

The frequency of gram-positive cocci $(51 \%)$ exceeded that of gram-negative bacilli $(40 \%)$ during the last 5-year period. CNS $(23 \%)$ moved past $P$. aeruginosa (14\%) into top place among the bacteremia pathogens. Although CNS, P. aeruginosa and $E$. coli remain among the top five pathogens, $K$. pneumoniae and $E$. cloacae have now been replaced by enterococci and streptococci. Such a pathogen shift may have reflected the common use of central venous catheters and second- and thirdgeneration cephalosporins $(1,4,13,14)$. Moreover, the empiric use of such effective antibiotics, often in association with rhG- 
CSF prophylaxis, may have lowered the frequency of gramnegative bacteremia from an occult undiagnosed infection.

$P$. aeruginosa belonged to the top two pathogens throughout the study period, with the frequency remaining almost unchanged (14\%-21\%). Also at other cancer centers in Japan, the organism remains as a major pathogen of bacteremia, despite prophylaxis with oral polymyxin-B or ciprofloxacin $(2,6,15)$. This is contrary to the decreasing frequency of $P$. aeruginosa infection at many cancer centers in the United States and Europe $(16,17)$. At the National Cancer Institute in USA, the frequency of $P$. aeruginosa bacteremia or infection inexplicably dropped to less than $1 \%$ of all episodes of fever and neutropenia in cancer patients in the last decade (16). Thus, the reason for such a discrepancy remains to be defined.

Although $E$. coli and $K$. pneumoniae usually originate in the alimentary canal (18), P. aeruginosa is mostly acquired exogenously as a mode of infection (1). Despite the use of newer $\beta$-lactam drugs with antipseudomonal activity, such as ceftazidime and imipenem, $P$. aeruginosa bacteremia continued to occur at a relatively constant frequency on our hematology ward. Indeed, all $P$. aeruginosa isolates obtained during the last 5 -year period proved susceptible to either of the drugs. Reducing the exogenous acquisition of the organism is, however, most urgent for infection prevention.

$P$. aeruginosa bacteremia is still associated with considerable morbidity and mortality in neutropenic patients with cancer $(2,5,6)$. Thus the flexibility in the choice of empiric antibiotics may well depend on the frequency of $P$. aeruginosa infection (16). On the other hand, the recent increase in the frequency of gram-positive bacteremia seems to justify the empiric use of vancomycin. In some randomized studies, however, no survival advantage was obtained from the addition of vancomycin to the current empiric regimens, such as ceftazidime plus amikacin $(3,19)$. Despite the shift toward gram-positive cocci, therefore, $p$. aeruginosa remains a key organism in considering the initial empiric antibiotic regimen.

\section{References}

1) Funada $H$, Machi $T$, Matsuda $T$. Bacteremia complicating acute leukemia with special reference to its incidence and changing etiological patterns. Jpn J Clin Oncol 18: 239-248, 1988.

2) Yokota $T$, Teshima H, Okajima $Y$, et al. Septicemia associated with hematopoietic disorders and its features according to respective primary disorders. Kansenshogaku Zasshi (J Jpn Assoc Infect Dis) 70: 108-115, 1996 (Abstract in English).
3) European Organization for Research and Treatment of Cancer (EORTC) International Antimicrobial Therapy Cooperative Group and the National Cancer Institute of Canada-Clinical Trials Group. Vancomycin added to empirical combination antibiotic therapy for fever in granulocytopenic cancer patients. J Infect Dis 163: 951-958, 1991 (published erratum appears in J Infect Dis 164: 832, 1991).

4) Bone RC. Gram-positive organisms and sepsis. Arch Intern Med 154: 2634, 1994.

5) Funada H, Machi T, Matsuda T. Pseudomonas aeruginosa bacteremia associated with hematologic disorders [III]. Prognostic factors. Kansenshogaku Zasshi (J Jpn Assoc Infect Dis) 63: 880-885, 1989 (Abstract in English).

6) Sakamoto M, Saruta K, Nakazawa Y, et al. Sepsis associated with hematological malignancies: prophylaxis of Pseudomonas aeruginosa sepsis. Kansenshogaku Zasshi (J Jpn Assoc Infect Dis) 70: 116-122, 1996.

7) Ohno R, Tomonaga M, Kobayashi T, et al. Effect of granulocyte colonystimulating factor after intensive induction therapy in relapsed or refractory acute leukemia. N Engl J Med 323: 871-877, 1990.

8) Funada $\mathrm{H}$, Teshima $\mathrm{H}$, Hattori $\mathrm{K}$. Total intestinal decontamination for prevention of infection in bone marrow transplantation. Jpn J Clin Oncol 13 Suppl 1: 111-126, 1983.

9) Takami A, Shiobara S. Opportunistic infection in bone marrow transplantation: current prevention in Kanazawa University Bone Marrow Transplantation Team. Ketsueki Shuyoka (Hematol Oncol) 33: 235-241, 1996 (in Japanese).

10) Emori TG, Gaynes RP. An overview of nosocomial infections, including the role of the microbiology laboratory. Clin Microbiol Rev 6: 428-442, 1993.

11) Mastrianni DM, Tung NM, Tenen DG. Acute myelogenous leukemia: current treatment and future directions. Am J Med 92: 286-295, 1992.

12) Ohno R. Therapy for leukemias. Nihon Naika Gakkai Zasshi (J Jpn Soc Intern Med) 86: 477-480, 1997 (in Japanese).

13) Yu VL. Enterococcal superinfection and colonization after therapy with moxalactam, a new broad-spectrum antibiotic. Ann Intern Med 94: 784785, 1981.

14) Kiehn TE, Armstrong D. Changes in the spectrum of organisms causing bacteremia and fungemia in immunocompromised patients due to venous access devices. Eur J Clin Microbiol Infect Dis 9: 869-872, 1990.

15) Sakamoto M. Optimal antibacterial chemotherapy for infectious diseases associated with hematological malignancies. Kansenshogaku Zasshi (J Jpn Assoc Infect Dis) 71: 72-82, 1997 (Abstract in English).

16) Pizzo PA. Management of fever in patients with cancer and treatmentinduced neutropenia. N Engl J Med 328: 1323-1332, 1993.

17) Ramphal R, Gucalp R, Rotstein C, Cimino M, Oblon D. Clinical experience with single agent and combination regimens in the management of infection in the febrile neutropenic patient. Am J Med 100 Suppl 6A: 83S-89S, 1996.

18) Funada H, Hattori K. Septicemia in acute leukemia. Jpn J Med 15: 115124, 1976.

19) Ramphal R, Bolger M, Oblon DJ, et al. Vancomycin is not an essential component of the initial empiric treatment regimen for febrile neutropenic patients receiving ceftazidime: a randomized prospective study. Antimicrob Agents Chemother 36: 1062-1067, 1992. 